\section{Citologia oncológica, captura \\ de híbridos II e inspeção visual \\ no rastreamento de lesões cervicais}

\author{
Pap smear, hybrid capture II, and visual inspection \\ in screening for uterine cervical lesions
}

Renata Clementino Gontijo 1,2 Sophie Françoise Mauricette Derchain 1 Eliana Borin Lopes Montemor 3 Luis Otávio Zanatta Sarian 1 Márcia Milena Pivatto Serra 4 Luiz Carlos Zeferino 1

Kari Juhani Syrjanen 5

\section{Introdução}

1 Faculdade de Ciências Médicas, Universidade Estadual de Campinas, Campinas, Brasil.

2 Faculdade de Medicina

de Jundiaí, Jundiai, Brasil.

3 Centro de Atenção Integral

à Saúde da Mulher.

Universidade Estadual de

Campinas, Campinas, Brasil.

4 Núcleo de Estudos

Populacionais, Universidade

Estadual de Campinas,

Campinas, Brasil.

5 Istituto Superiore

di Sanità, Roma, Itália.

Correspondência

R. C. Gontijo

Departamento de

Tocoginecologia, Faculdade

de Ciências Médicas,

Universidade Estadual

de Campinas. Rua Alexander

Fleming 101, Campinas, SP

13083-970, Brasil.

rgontijo@terra.com.br

\begin{abstract}
The objective of this study was to evaluate alter-
Abstract ations in Pap smear, hybrid capture II (HCII), and visual inspection with acetic acid (VIA) in 684 women treated at a primary health care unit. The performance and agreement of the exams were evaluated. The study also described social, demographic, and reproductive factors and their association with uterine cervical lesions. Women had specimens taken for Pap smear, HCII, and VIA. When at least one of the tests was positive, colposcopy was performed and targeted biopsies were taken from any suspicious lesions. Performance of tests was evaluated. Women's distribution in relation to social, demographic, and reproductive factors and histological diagnosis was evaluated using the odds ratio. Among 198 women with at least one positive screening test, only 21 showed histological disease. Sensitivities of the tests were similar. VIA and Pap smear presented higher specificity than HCII. Only absence of a previous Pap smear was associated with the presence of histological disease. Pap smear performed better than VIA and HC II. Absence of previous cytology was associated with histological disease.
\end{abstract}

Cytology; Cervical Intraepithelial Neoplasia; Human Papillomavirus
A maioria dos casos de câncer do colo uterino pode ser prevenida por meio do rastreamento. A citologia oncológica é o principal método utilizado no diagnóstico precoce das lesões cervicais. Em locais onde a qualidade, cobertura e seguimento do rastreamento citológico são elevados, a incidência do câncer cervical foi reduzida em até $80 \% 1,2$.

Os resultados de meta-análises sugerem que o rastreamento citológico tem grande variação de sensibilidade para detectar lesões histológicas 3 . Uma característica do exame citopatológico é que predomina o trabalho manual, desde a colheita do material até a emissão e liberação do resultado pelo laboratório. O desempenho pode, assim, estar relacionado com a qualidade dos recursos humanos envolvidos 1,4 .

Métodos alternativos e tecnologicamente diferentes da citologia oncológica têm sido sugeridos para o rastreamento do câncer cervical, entre estes a inspeção visual com ácido acético (IVA) e os testes para detecção de infecção pelo papilomavírus humano (HPV) por intermédio da biologia molecular 2 . A IVA tem recebido atenção considerável como técnica alternativa para países em desenvolvimento 5,6. A cérvice é lavada com ácido acético a $3 \%$ ou a $5 \%$ e observada a olho nu a procura de áreas aceto-brancas. Este método é simples, requer mínima infra-estrutura e poucos equipamen- 
tos; também podendo ser realizado por pessoal paramédico ou enfermeiros, em unidades básicas de saúde $1,4,5,6$.

Paralelamente, com o conhecimento do papel do HPV na promoção da neoplasia cervical, verificou-se que o uso dos testes de detecção de DNA-HPV poderia ser de utilidade como método de rastreamento no diagnóstico precoce de lesões cervicais 7 . A captura de híbridos II é um procedimento de hibridização molecular, de processamento rápido e leitura confiável para detectar 18 tipos de HPV divididos em grupos de baixo $(6,11,42,43$ e 44$)$, e de alto risco oncogênico $(16,18,31,33,35,39,45,51,52,56$, 58, 59 e 68) 7 . Os testes de detecção do HPV associados à citologia podem ser úteis na identificação de mulheres de risco para lesões cervicais mais graves e também para evitar colposcopias desnecessárias 1 .

Mais de 25 anos de pesquisas epidemiológicas estabeleceram a forte associação entre a atividade sexual e desenvolvimento do carci noma cervical, o que implicou um agente microbiológico como seu fator etiológico primário ${ }^{8}$. Paralelamente, pesquisas com métodos de biologia molecular evidenciaram que este agente é o HPV, aceito atualmente como a causa de quase $100 \%$ das neoplasias cervicais 9,10 . Entretanto, nem todas as mulheres infectadas pelo HPV progredirão para câncer e mesmo as lesões intra-epiteliais cervicais podem apresentar regressão espontânea. Alguns fatores têm sido estudados como associados à persistência da infecção pelo HPV ou às lesões cervicais morfológicas. Como várias características do comportamento sexual são consistentemente associadas com um risco maior para o câncer cervical, podemos considerar que este câncer se comporta como uma doença sexualmente transmissível. Múltiplos parceiros sexuais, parceiros sexuais promíscuos e início da atividade sexual precoce, além do nível sócio-econômico baixo, alta paridade, tabagismo, uso de anticoncepcional hormonal, fatores dietéticos e imunossupressão têm sido implicados como potenciais fatores de risco para o carcinoma cervical 11,12 .

Por outro lado, embora os exames de rastreamento sejam capazes de selecionar as mulheres com maior risco de apresentar uma lesão cervical pré-neoplásica ou mesmo invasora, nem sempre um resultado alterado corresponde à doença histológica. Observa-se que o valor preditivo positivo (VPP) dos testes na detecção de lesão histológica é sempre baixo, mesmo que variável. A capturas de híbridos II apresenta VPP entre $14 \%$ e $23 \%$, o VPP da citologia oncológica varia entre $23 \%$ e $33 \%$, enquanto a inspeção visual com ácido acético (IVA) apresenta os menores VPP, variando entre $11 \%$ e $19 \% 5,7,13$.

Desse modo, o objetivo deste estudo foi avaliar a presença de alterações citológicas, a detecção de DNA-HPV e a presença de anor malidades cervicais à IVA em mulheres que demandam assistência ginecológica em uma unidade de saúde, assim como avaliar o desempenho e a concordância entre os exames na detecção de lesões pré-neoplásicas e neoplásicas cervicais. Também se procurou a descrição das características sócio-demográficas e reprodutivas destas mulheres e avaliar sua associação com a presença de doença histológica.

\section{Material e métodos}

Para este estudo de corte transversal, foram avaliadas 684 mulheres atendidas no Centro de Saúde Santa Bárbara, na cidade de Campinas, São Paulo, entre fevereiro e dezembro de 2002. Foram incluídas mulheres com idade entre $18 \mathrm{e}$ 60 anos. As mulheres com antecedente de imunossupressão ou que apresentassem resultado de citologia oncológica prévia anormal foram excluídas. Este estudo foi aprovado pelo Comitê de Ética em Pesquisa da Faculdade de Ciências Médicas da Universidade Estadual de Campinas (FCM, UNICAMP) e pela Comissão Nacional de Ética em Pesquisa (CONEP), em outubro de 2001, processo número: 25000079145/2001-53.

Após assinarem o termo de consentimento livre e esclarecido, as participantes responderam a um questionário referente aos fatores sócio-demográficos e reprodutivos descritos a seguir: idade, referida pela mulher em anos completos no momento da consulta; se a mulher vivia com seu companheiro ou não; escolaridade em anos completos de estudo formal; idade ao início da atividade sexual, número total de parceiros sexuais, bem como número de parceiros nos últimos doze meses. Também foi avaliado o número total de partos normais ou operatórios e se a mulher estava ou não grávida no momento do exame. Indagou-se sobre a utilização de método contraceptivo hormonal e se fumava ou não no momento da consulta. Finalmente, a mulher indicava se já havia coletado alguma citologia oncológica prévia em qual quer momento de sua vida.

A seguir todas as mulheres foram submetidas seqüencialmente à coleta de material para citologia oncológica, capturas de híbridos II para detecção do DNA-HPV de alto risco oncogênico (tipos $16,18,31,33,35,39,45,51,52,56,58,59,68$ ) 7 e IVA, após aplicação de ácido acético a 5\%. 
O esfregaço citológico foi constituído de duas amostras, representativas do raspado da ectocérvice e do escovado da endocérvice. As lâminas foram coradas pelo método de Papanicolau e foram avaliadas com base no Sistema de Bethesda 14. Os resultados citológicos foram classificados em: (1) alterações inflamatórias; (2) lesões escamosas pré-invasoras, incluindo atipias de células escamosas de origem indeterminada (ASCUS); (3) lesões escamosas de baixo grau (NIC 1 e HPV); (4) lesões escamosas de alto grau (NIC 2 e 3); (5) atipias glandulares préinvasoras, incluindo atipias glandulares de origem indeterminada (AGUS) e adenocarcinoma in situ; e finalmente (6) carcinoma invasor escamoso ou glandular.

Para análise de DNA-HPV, utilizou-se o teste de capturas de híbridos II (Digene do Brasil Ltda.), obtendo-se material com a utilização de uma escova endocervical. Para classificar o resultado e quantificar a carga viral, utilizou-se um valor de corte diário, e amostras com emissão de luz maior que o ponto de corte foram consideradas positivas e aquelas com emissão de luz menor foram consideradas negativas. Uma unidade relativa de luz (URL) corresponde a $1 \mathrm{pg} / \mathrm{ml}$ de DNA-HPV, equivalente a 0,1 cópia de vírus/célula.

Após a coleta da citologia oncológica e da captura de híbridos II, foi aplicado ácido acético a $5 \%$ no colo. Depois de um minuto, o colo foi iluminado com uma lâmpada elétrica de 100 Watts e examinado a olho nu à procura de áreas aceto-brancas. A aparência visual foi classificada segundo o Atlas de Inspeção Visual da Cérvice 15 em: normal, sugestivo de neoplasia intraepitelial e sugestivo de câncer.

Após a coleta dos exames, o retorno para resultados foi marcado em 60 dias e as mulheres que compareceram para a segunda consulta e apresentavam pelo menos um exame com resultado positivo foram submetidas à colposcopia. As imagens colposcópicas encontradas foram classificadas segundo a Nomenclatura Internacional dos Achados Colposcópicos 16 em: achados colposcópicos normais, anormais, suspeita de câncer invasor e achados insatisfatórios. Quando a colposcopia apresentou resultado anormal, foram realizadas biopsias, utilizando-se pinças de saca-bocado, sendo retiradas amostras das regiões alteradas.

Para análise estatística, inicialmente foram elaboradas tabelas descritivas, com o resultado dos exames de rastreamento e o resultado histológico. O desempenho dos exames envolveu o cálculo da sensibilidade, especificidade e valores preditivos positivo e negativo, usando IC95\%. O padrão ouro foi assim definido: as mulheres com todos os exames negativos não foram submetidas à colposcopia e foram consideradas sem doença. Da mesma forma, sempre que a colposcopia foi realizada e apresentou resultado normal, ou quando a biopsia apresentou resultado cervicite ou metaplasia foram consideradas com diagnóstico final negativo. As mulheres cujo diagnóstico histológico foi compatível com NIC 1, 2, 3 ou câncer invasor foram consideradas com diagnóstico final positivo. As mulheres com pelo menos um resultado de exame de rastreamento (citologia oncológica, captura de híbridos II ou IVA) positivo que não compareceram para colposcopia foram classificadas como "exame positivo não verificado" e foram excluídas da análise de desempenho.

Por fim, foram elaboradas tabelas descritivas com a distribuição das mulheres de acordo com suas características sócio-demográficas e reprodutivas. Para avaliar possíveis associações entre essas variáveis e a presença de doença, foram utilizados os testes do qui-quadrado com correção de Yates quando necessário, avaliando como significativo um valor de $\mathrm{p}<0,05$.

\section{Resultados}

Podemos verificar na Tabela 1 que quase $90,0 \%$ das mulheres apresentaram resultado de citologia normal. Setenta e uma $(10,4 \%)$ apresentaram atipias celulares, sendo 50 (7,3\%) ASCUS, um $(0,1 \%)$ AGUS, 15 (2,2\%) alterações escamosas de baixo grau (HPV/NIC 1$)$ e cinco $(0,7 \%)$ alterações escamosas de alto grau (NIC 2 ou NIC 3). Não foi encontrada nenhuma citologia oncológica sugestiva de câncer. Observamos também que em aproximadamente $20,0 \%$ das mulheres foi detectado o DNA-HPV. Em relação à IVA, $8 \%$ das mulheres apresentaram o resultado positivo.

Na Tabela 2, verificamos que das 198 (29\%) mulheres com pelo menos um exame alterado e que foram referidas para colposcopia, 48 não compareceram. Entre as 150 mulheres com pelo menos um exame alterado e que fizeram colposcopia, 91 não apresentaram imagem suspeita e não foram submetidas à biopsia. Por outro lado, das 59 mulheres que foram biopsiadas, os resultados histológicos foram compatíveis com cervicite/metaplasia escamosa em 38, HPV/NIC 1 em dez, NIC 2 em oito, NIC 3 em duas e carcinoma invasor em uma, totalizando 21 mulheres com lesão histológica cervical.

Na Tabela 3, encontramos que a citologia oncológica e a captura de híbridos II apresentaram a sensibilidade $(67 \%)$ maior do que a IVA (52\%), por mais que esta diferença não fosse 
Tabela 1

Distribuição das mulheres segundo o resultado dos exames.

\begin{tabular}{lrr}
\hline Exame & $\mathbf{n}$ & $\%$ \\
\hline Citologia oncológica & 613 & 89,6 \\
$\quad$ Normal & 50 & 7,3 \\
Atipias de células escamosas de origem indeterminada & 15 & 2,2 \\
Lesão escamosa de baixo grau & 5 & 0,7 \\
Lesão escamosa de alto grau & 1 & 0,1 \\
Atipias glandulares de origem indeterminada & & \\
& & \\
Captura de híbridos II & 553 & 80,3 \\
Negativa < 1 unidade relativa de luz & 131 & 19,2 \\
Positiva 1 unidade relativa de luz & & \\
Inspeção visual com ácido acético & 629 & 92,0 \\
Negativa & 55 & 8,0 \\
Positiva & & \\
\hline
\end{tabular}

Tabela 2

Distribuição das mulheres segundo o diagnóstico final.

\begin{tabular}{lrr}
\hline Diagnóstico & $\mathbf{n}$ & $\%$ \\
\hline Sem colposcopia todos os exames negativos & 486 & 71,0 \\
Sem colposcopia com pelo menos um exame positivo & 48 & 7,0 \\
Com colposcopia por exame positivo sem biopsia & 91 & 13,3 \\
Com colposcopia por exame positivo com biopsia & 59 & 8,7 \\
Cervicite/metaplasia & 38 & 64,4 \\
HPV/NIC 1 & 10 & 16,9 \\
NIC 2 & 8 & 13,6 \\
NIC 3 & 2 & 3,4 \\
Câncer invasor & 1 & 1,7 \\
Total & 684 & \\
\hline
\end{tabular}

$\mathrm{NIC}=$ Lesões escamosas

Tabela 3

Desempenho dos três exames.

\begin{tabular}{lccc}
\hline Desempenho & CO (IC95\%) & CH II (IC95\%) & IVA (IC95\%) \\
\hline Sensibilidade & $67(47-87)$ & $67(47-87)$ & $52(31-74)$ \\
Especificidade & $66(58-74)$ & $31(23-39)$ & $79(72-86)$ \\
VPP & $24(13-35)$ & $14(7-20)$ & $29(15-43)$ \\
VPN & $92(87-98)$ & $85(75-95)$ & $91(86-96)$ \\
\hline
\end{tabular}

$\mathrm{VPP}=$ valor preditivo positivo; VPN = valor preditivo negativo; $\mathrm{CO}=$ citologia oncológica; $\mathrm{CH}$ II = captura de híbridos; IVA = inspeção visual com ácido acético significativa. As especificidades da IVA (79\%) e da citologia oncológica (66\%) isoladamente foram significativamente maiores que da captura de híbridos II (31\%). Os valores preditivos negativos dos três testes foram semelhantes e altos. Já os valores preditivos positivos também foram semelhantes, ainda que baixos.

Na Tabela 4, comparando os resultados da citologia oncológica, da captura de híbridos II e da IVA, observamos que entre as mulheres com resultado de citologia oncológica negativo, a grande maioria apresentou também o colo normal à inspeção visual, embora em 94 tenha sido detectado o DNA viral. Entretanto, 33 mulheres com resultados de citologia oncológica e captura de híbridos II negativos apresentaram alguma alteração na inspeção visual do colo. Dentre as mulheres com resultado de citologia oncológica positivo, a maior parte apresentou o colo normal a IVA, mesmo que tenha sido detectado o DNA-HPV em mais da metade destas mulheres. Apenas sete mulheres apresentaram os três exames positivos, e, por outro lado, 486 mulheres apresentaram todos os três exames negativos.

Observamos, na Tabela 5, que mais de $60 \%$ das mulheres avaliadas tinham menos de 35 anos, eram casadas, estudaram mais que quatro anos e iniciaram sua atividade sexual com menos de 18 anos. A grande maioria teve menos que cinco parceiros sexuais durante toda a vida e somente um parceiro nos últimos doze meses. Um pouco mais que a metade da população estudada teve até dois partos, a maioria não estava grávida e não usava método anticoncepcional hormonal. Quinze porcento da mulheres nunca tinham feito citologia oncológica prévia e cerca de $21 \%$ eram fumantes.

Na Tabela 6, no entanto, quando comparamos os fatores sócio-demográficos e reprodutivos com o diagnóstico histológico, apenas o fato de nunca ter sido submetida ao exame de rastreamento citológico previamente esteve significativamente associado com a presença de doença histológica. Como nenhuma outra variável apresentou valor de $\mathrm{p}<0,1$ não foi possível realizar análise de regressão logística.

\section{Discussão}

O câncer de colo do útero é problema de saúde pública em muitas regiões do mundo, apesar de a tecnologia para seu diagnóstico precoce estar disponível há muitas décadas. A proposta de um programa de rastreamento é, além de diagnosticar o câncer em estádios iniciais, detectar e remover lesões de alto grau prevenindo 
Comparação dos resultados dos exames em 684 mulheres.

\begin{tabular}{lcc}
\hline & \multicolumn{2}{c}{ IVA } \\
& Negativa & Positiva \\
\hline CO negativa & & \\
CH II negativa (<1URL) & 486 & 33 \\
CH II positiva (> 1URL) & 82 & 12 \\
CO positiva & & \\
CH II negativa (<1URL) & 31 & 3 \\
CH II positiva (> 1URL) & 30 & 7 \\
Total & 629 & 55 \\
\hline
\end{tabular}

$\mathrm{CO}=$ citologia oncológica; $\mathrm{CH}$ II = captura de híbridos; IVA = inspeção visual com ácido acético; URL = unidade relativa de luz.

sua potencial progressão para carcinoma 17 . Geralmente, tais programas são baseados em citologia e sua desvantagem é que requerem laboratórios estabelecidos e citologistas e citotécnicos bem treinados 4 . Por este motivo, métodos opcionais para rastreamento têm sido descritos atualmente. Existe um substancial interesse no uso dos testes de biologia molecular baseado na premissa de que a detecção do HPV poderia apresentar um desempenho diagnóstico aceitável sendo melhor reproduzível e mais facilmente adaptado para a prática clínica que a citologia convencional 17 .

Porém, o papel da detecção do DNA-HPV no rastreamento é controverso. Em um estudo realizado com 2.098 mulheres usando o teste de detecção de HPV como método de rastreamento, Ratnam et al. 18 encontraram 10,8\% dos resultados positivos. Já Denny et al. 19 encontraram taxa de positividade de $16,2 \%$. Nosso estudo mostrou uma taxa de positividade de $19,2 \%$ entre as mulheres submetidas ao exame. Este resultado pode ser justificado pelo fato de que mais de $60 \%$ das mulheres estudadas terem a idade menor do que 35 anos. Sabe-se que nesta faixa etária as infecções subclínicas pelo HPV são comuns e que a grande maioria das mulheres tem infecção transitória que, em curto período de tempo, entra em equilíbrio com o seu sistema imunológico sem resultar em doença 2,17 . A especificidade da capturas de híbridos II nesta amostra foi baixa (31\%), resultando em maior número de resultados falsopositivos. A baixa especificidade da captura de híbridos II em mulheres com idade inferior a 30 anos é um obstáculo à sua implementação
Tabela 5

Distribuição das mulheres segundo as variáveis sócio-demográficas e reprodutivas.

\begin{tabular}{|c|c|c|}
\hline Característica & n & $\%$ \\
\hline \multicolumn{3}{|l|}{ Idade (anos) } \\
\hline$\geq 35$ & 258 & 38 \\
\hline$<35$ & 426 & 62 \\
\hline \multicolumn{3}{|c|}{ Vive com companheiro } \\
\hline Sim & 483 & 71 \\
\hline Não & 201 & 29 \\
\hline \multicolumn{3}{|c|}{ Escolaridade (anos de estudo) } \\
\hline$\leq 4$ & 204 & 30 \\
\hline$>4$ & 480 & 70 \\
\hline \multicolumn{3}{|c|}{ Início da atividade sexual (anos) } \\
\hline$\geq 18$ & 276 & 40 \\
\hline$<18$ & 408 & 60 \\
\hline \multicolumn{3}{|c|}{ Parceiros sexuais durante a vida } \\
\hline$<5$ & 613 & 90 \\
\hline$\geq 5$ & 71 & 10 \\
\hline \multicolumn{3}{|c|}{ Parceiros sexuais nos últimos 12 meses } \\
\hline$\leq 1$ & 648 & 95 \\
\hline$>1$ & 36 & 5 \\
\hline \multicolumn{3}{|c|}{ Número total de partos } \\
\hline$\geq 2$ & 371 & 54 \\
\hline$<2$ & 313 & 46 \\
\hline \multicolumn{3}{|l|}{ Grávida } \\
\hline Não & 638 & 93 \\
\hline Sim & 46 & 7 \\
\hline \multicolumn{3}{|c|}{ Anticoncepcional oral hormonal } \\
\hline Não & 469 & 69 \\
\hline $\operatorname{Sim}$ & 215 & 31 \\
\hline \multicolumn{3}{|c|}{ Citologia prévia } \\
\hline Sim & 584 & 85 \\
\hline Não & 100 & 15 \\
\hline \multicolumn{3}{|c|}{ Tabagista atual } \\
\hline Não fuma & 540 & 79 \\
\hline Fuma & 140 & 21 \\
\hline
\end{tabular}

como substituto da citologia oncológica nos programas de rastreamento do câncer do colo uterino. Testes de triagem devem apresentar um alto valor preditivo positivo. Uma alta taxa de resultados falso-positivos leva à repetição de exames, com conseqüente aumento do custo, ansiedade da mulher e perda de tempo. Há que se contar com o inconveniente de consul- 
Variáveis sócio-demográficas e reprodutivas e o diagnóstico final em análise univariada.

\begin{tabular}{|c|c|c|c|c|c|}
\hline \multirow[t]{2}{*}{ Característica } & \multicolumn{2}{|c|}{ Sem doença } & \multicolumn{2}{|c|}{ Com doença } & \multirow[t]{2}{*}{$\mathbf{p}$} \\
\hline & $\mathrm{n}$ & $\%$ & $\mathrm{n}$ & $\%$ & \\
\hline \multicolumn{6}{|l|}{ Idade (anos) } \\
\hline$\geq 35$ & 237 & 38 & 6 & 29 & \\
\hline$<35$ & 378 & 62 & 15 & 71 & 0,48 \\
\hline \multicolumn{6}{|c|}{ Vive com companheiro } \\
\hline $\operatorname{Sim}$ & 443 & 72 & 13 & 62 & \\
\hline Não & 172 & 28 & 8 & 38 & 0,44 \\
\hline \multicolumn{6}{|c|}{ Escolaridade (anos de estudo) } \\
\hline$\leq 4$ & 187 & 31 & 5 & 24 & \\
\hline$>4$ & 428 & 69 & 16 & 76 & 0,94 \\
\hline \multicolumn{6}{|c|}{ Início da atividade sexual (anos) } \\
\hline$\geq 18$ & 255 & 42 & 7 & 33 & \\
\hline$<18$ & 360 & 58 & 14 & 67 & 0,60 \\
\hline \multicolumn{6}{|c|}{ Parceiros sexuais durante a vida } \\
\hline$<5$ & 554 & 90 & 17 & 81 & \\
\hline$\geq 5$ & 61 & 10 & 4 & 19 & 0,32 \\
\hline \multicolumn{6}{|c|}{ Parceiros sexuais nos últimos 12 meses } \\
\hline$\leq 1$ & 584 & 95 & 19 & 90 & \\
\hline$>1$ & 31 & 5 & 2 & 10 & 0,68 \\
\hline \multicolumn{6}{|c|}{ Número total de partos } \\
\hline$\geq 2$ & 336 & 55 & 8 & 38 & \\
\hline$<2$ & 279 & 45 & 13 & 62 & 0,20 \\
\hline \multicolumn{6}{|l|}{ Grávida } \\
\hline Não & 574 & 93 & 21 & 100 & \\
\hline Sim & 41 & 7 & 0 & 0 & - \\
\hline \multicolumn{6}{|c|}{ Anticoncepcional oral hormonal } \\
\hline Não & 423 & 69 & 15 & 71 & \\
\hline Sim & 192 & 31 & 6 & 29 & 0,98 \\
\hline \multicolumn{6}{|c|}{ Citologia prévia } \\
\hline Sim & 530 & 86 & 14 & 67 & \\
\hline Não & 85 & 14 & 7 & 33 & 0,02 \\
\hline \multicolumn{6}{|c|}{ Tabagismo atual } \\
\hline Não fuma & 491 & 80 & 14 & 67 & \\
\hline Fuma & 124 & 20 & 7 & 33 & 0,23 \\
\hline
\end{tabular}


tas repetidas com procedimentos eventualmente desnecessários.

Em lugares onde programas de rastreamento baseados em citologia não são adequados, a IVA tem recebido atenção como uma técnica para redução das taxas de câncer cervical 6 . Neste estudo, a IVA apresentou resultado positivo em $8 \%$ das mulheres enquanto que a citologia esteve alterada em 10,4\%, mostrando resultados semelhantes aos da literatura. Sankaranarayanan et al. 20 avaliaram 3 mil mulheres e encontraram 298 (9,9\%) IVA alteradas e 307 (10,2\%) citologias positivas. Estudos conduzidos na Índia, África e China mostraram um desempenho da IVA melhor ou igual ao da citologia na correta identificação de doenças; todavia, com especificidade consistentemente menor que a citologia 5,6,20. Em nosso estudo, embora aparentemente a sensibilidade da IVA fosse menor que a da citologia oncológica, e sua especificidade fosse maior, estas diferenças não foram estatisticamente significativas. Estes resultados divergentes podem ser explicados pela qualidade da cobertura citológica da nossa região, mostrando melhor desempenho da citologia oncológica na detecção de casos positivos.

Para algumas regiões, os programas de rastreamento baseados nas alterações visuais do colo têm vantagens significativas sobre aqueles baseados em citologia e/ou nos testes para detecção de HPV: são baratos, de simples realização e, por apresentarem resultado imediato, minimizam as perdas de seguimento e eventualmente permitem avaliação e tratamento em uma só consulta 4,20 . Sankaranarayanan et al. 4 também advogam pelo tratamento realizado com cauterização ou crioterapia na primeira visita, logo após o resultado da IVA. Referem que os custos diminuem com o tratamento em uma única consulta e que o tratamento das mulheres sem doença devido a um resultado falso-positivo pode ser considerado como "um preço aceitável a pagar pelo efetivo controle do carcinoma cervical". Considerando, na nossa casuística, o valor preditivo positivo da IVA de apenas $29 \%$, o tratamento imediato nesta população teria levado a um número excessivo de cauterizações em mulheres sadias. No presente estudo, após a coleta dos exames, a mulher teve seu retorno agendado para resultado. Quando um exame mostrou resultado positivo, ela foi encaminhada e submetida à colposcopia. Observamos que 48 das 198 mulheres com pelo menos um exame alterado não voltaram para a colposcopia, o que poderia justificar a aplicação de tratamento imediato mesmo que, para muitas das mulheres, esta conduta pudesse ser considerada excessiva. Por outro lado, uma mulher apresentou câncer e, neste caso, a cauterização no momento da primeira consulta sem avaliação histopatológica teria sido incorreta. Assim, é importante salientar que o tratamento imediato pode ser visto como uma opção ética e racional aceitável somente nas situa ções em que a infra-estrutura é pobre e os recursos são limitados 21 , fato que não se aplica à unidade de saúde onde foi realizado o estudo.

Vários fatores sócio-demográficos e reprodutivos são classicamente descritos como fatores de risco para lesões cervicais. Segundo Brito et al. 22 , o risco de neoplasia cervical está relacionado à idade da primeira relação sexual e múltiplos parceiros, indicando que o coito precoce pode aumentar a sensibilidade da cérvice aos efeitos de um agente sexualmente transmitido. Em estudo recente realizado com mulheres de 15 a 29 anos no Acre, as alterações celulares epiteliais estiveram associadas ao maior número de parceiros, baixa escolaridade, tabagismo e história de doença sexualmente transmissível. A freqüência de lesões precursoras em mulheres de faixa etária mais jovem evidenciou a exposição precoce aos fatores de risco que antecipa o desenvolvimento do câncer do colo uterino 23 .

O tabagismo é tido por alguns autores como fator independentemente associado à presença de lesões pré-neoplásicas cervicais de alto grau e carcinoma invasor 11,24,25. Também mulheres usuárias de contraceptivos hormo nais apresentam maior risco para carcinoma cervical, o que sugere que o fator hormonal pode desempenhar um papel de co-fator na condição de portadoras crônicas de HPV ou na progressão das neoplasias relacionadas ao HPV 9,26. O uso de contraceptivo hormonal oral por menos que cinco anos não parece ser relacionado ao câncer cervical, embora o risco aumente significativamente em usuárias por mais de cinco a dez anos 27 .

Apenas o fato de nunca ter coletado citologia oncológica previamente esteve significati vamente associado com a presença de doença na avaliação histológica. Este resultado evidencia a importância dos programas de rastrea mento baseados em citologia na detecção de doença do colo uterino. Uma proteção significativa é observada em mulheres que já fizeram pelo menos um exame em toda vida, e o risco cai quando o número de exames realizados é de seis ou mais 26 . Aproximadamente $50 \%$ dos cânceres nos Estados Unidos da América ocorrem em mulheres nunca antes rastreadas e $10 \%$ acontecem naquelas não examinadas nos últimos cinco anos 17. Isso posto, talvez o maior ganho na redução de incidência e mortalidade 
pelo câncer do colo do útero esteja em aumentar as taxas de rastreamento, independentemente dos fatores de risco, entre mulheres que nunca fizeram o exame ou o fazem com pouca freqüência.

Deste modo, é importante ser enfatizado que o rastreamento citológico é somente uma parte da abordagem para o controle do câncer do colo do útero. A utilização de técnicas alternativas, como a detecção do DNA-HPV e a inspeção visual com ácido acético associados à ci-

\section{Resumo}

Avaliar as alterações na citologia oncológica, na captura híbrida II e na inspeção visual com ácido acético (IVA) a 5\% em 684 mulheres atendidas em uma unidade de saúde, e avaliar o desempenho e a concordância entre os exames. Procurar descrever as características sócio-demográficas e reprodutivas (SDR) destas mulheres e sua associação com a presença de doença. As mulheres foram submetidas à coleta de citologia, captura e IVA, e as com exame positivo foram submetidas à colposcopia com biopsia, se necessário. Foi calculado o desempenho dos testes e a associação dos fatores SDR com o diagnóstico histológico foi avaliada por intermédio do cálculo do odds ratio. Embora 198 mulheres apresentassem pelo menos um teste alterado, apenas 21 apresentaram lesão histológica. A sensibilidade dos testes foi semelhante enquanto as especificidades da IVA e da citologia foram maiores que a da captura híbrida II. Apenas a ausência de citologia esteve associada à presença de doença. O desempenho da citologia foi maior que o da IVA, que foi maior que o da captura. A ausência de citologia foi associada com doença histológica.

Citologia; Neoplasia Intra-epitelial Cervical; Papillomavirus Humano tologia poderiam resultar em melhoria da detecção das lesões cervicais, com conseqüente diminuição da incidência e mortalidade por esta doença. Uma melhor orientação das mulheres por programas que envolvam aspectos de educação sexual poderia potencialmente prevenir infecções pelo HPV e suas conseqüências. A conscientização dessas mulheres quanto à importância de ser rastreada pelo menos uma vez na vida deve estar também entre os nossos objetivos.

\section{Colaboradores}

R. C. Gontijo participou da coleta de dados e preparação do artigo. S. F. M. Derchain colaborou na orientação da pesquisa e preparação do artigo. E. B. L. Montemor colaborou na leitura das lâminas de citologia. L. O. Z. Sarian contribuiu com o banco de dados. M. M. P. Serra participou da análise estatística. L. C. Zeferino e K. J. Syrjanen contribuíram na preparação do artigo.

\section{Agradecimentos}

Aos órgãos financiadores: Comitê Europeu de Pesquisa da Comunidade Econômica Européia INCO DEV 4-CT-2001-(10013). 


\section{Referências}

1. Mandelblatt J, Lawrence W, Womack SM, Jacobson D, Yi B, Hwang Y, et al. Benefits and costs of using HPV testing to screen for cervical cancer. JAMA 2002; 287:2372-81.

2. Syrjänen KJ, Syrjänen SM. Papillomavirus infections in human pathology. Chichester: John Wiley \& Sons; 2000.

3. Bastian L, Datta S, Hasselblad V, Hickey J, Myers E, Nanda K. Evidence report - evaluation of cervical cytology. Rockville: Agency for Health Care Policy and Research; 1999. (AHCPR Publication no 99-E010).

4. Sankaranarayanan R, Buduck AM, Rajkumar R. Effective screening programs for cervical cancer in low and middle-income developing countries. Bull World Health Organ 2001; 79:954-62.

5. Belinson J, Pretorius R, Zhang WZ, Wu LY, Qiao YL, Elson P. Cervical cancer screening study by simple visual inspection after acetic acid. Obstet Gynecol 2001; 98:441-4.

6. Anonimous. Visual inspection with acetic acid for cervical cancer screening: test qualities in a primary-care setting. Lancet 1999; 353:869-73.

7. Clavel C, Masure M, Bory JP, Putaud I, Mangeonjean C, Lorenzato M, et al. Human papillomavirus testing in primary screening for detection of highgrade cervical lesions: a study of 7932 women. $\mathrm{Br}$ J Cancer 2001; 89:1616-22.

8. Franco EL, Villa LL, Ruiz A, Costa MC. Transmission of cervical papillomavirus infection by sexual activity: differences between low and high oncogenic risk types. J Infect Dis 1995, 172:756-63.

9. Bosch FX, Munõz N, Sanjosé S. Human papillomavirus and other risk factors for cervical cancer. Biomed Pharmacother 1997; 51:268-75.

10. Walboomers JM, Jacobs MV, Manos MM, Bosch FX, Kummer JÁ, Shah KV, et al. Human papillomavirus is a necessary cause of invasive cervical cancer worldwide. J Pathol 1999; 189:12-9.

11. Kjellberg L, Hallmans G, Åhren AM, Johansson R, Bergman F, Wadell G, et al. Smoking, diet, pregnancy, and oral contraceptive use as risk factors for cervical intra-epithelial neoplasia in relation to human papillomavirus infection. Br J Cancer 2000; 82:1332-8.

12. Syrjänen KJ, Syrjänen SM. Human papillomavirus (HPV) typing as an adjunct to cervical cancer screening. Cytopathology 1999; 10:8-15.

13. Blumenthal P, Gaffikin L, Chirenje ZM, Mcgrath J, Womack S, Shah K. Adjunctive testing for cervical cancer in low resource settings with visual inspection, HPV and Pap smear. Int J Gynaecol Obstet 2001; 72:47-53.

14. Kurman RJ, Solomon D. The Bethesda System for reporting cervical/vaginal cytologic diagnoses. New York: Springer-Verlag; 1994.

15. Blumenthal P. Atlas for unaided visual inspection of the cervix. Baltimore: JHPIEGO; 1997.
16. Stafl A, Wilbanks GD. An international terminology of colposcopy: report of the Nomenclature Committee of the International Federation of Cervical Pathology and Colposcopy. Obstet Gynecol 1991, 77:313-4.

17. Saslow D, Runowicz C, Solomon D, Moscicki AB, Smith RA, Eyre H, et al. American Cancer Society guideline for the early detection of cervical neoplasia and cancer. CA Cancer J Clin 2002; 52:34262.

18. Ratnam S, Franco E, Ferenczy A. Human papillomavirus testing for primary screening of cervical cancer precursors. Cancer Epidemiol Biomarkers Prev 2000; 9:945-51.

19. Denny L, Kuhn L, Pollack A, Wainwright H, Wright T. Evaluation of alternative methods of cervical cancer screening for resource-poor settings. Cancer 2000; 89:826-33.

20. Sankaranaranayan R, Wesley R, Somanathan $T$, Dhakad N, Shyamalakumary B, Amma S. Visual inspection of the uterine cervix after the application of acetic acid in the detection of cervical carcinoma and its precursors. Cancer 1998, 83:2150-6.

21. Goldie SJ, Kuhn L, Denny L, Pollack A, Wright TC. Policy analysis of cervical cancer screening strategies in low-resource settings: clinical benefits and cost-effectiveness. JAMA, 2001; 285:3107-15.

22. Brito NMB, Moreira SFS, Ferreira MA, Lopes RV Bastos AAC. Aspectos epidemiológicos das neoplasias intra-epiteliais cervicais identificadas por citologia oncótica. Rev Para Med 2000, 14:42-6.

23. Leal EAS, Leal Jr. OS, Guimarães MH, Vitoriano MN, Nascimento TL, Costa OLN. Lesões precursoras do câncer de colo em mulheres adolescentes e adultas jovens do Município de Rio Branco Acre. Rev Bras Ginecol Obstet 2003; 25:81-6.

24. Derchain SFM, Roteli-Martins CM, Syrjänen KJ, Abreu HI, Martinez EZ, Alves VAF. Association of oncogenic human papillomavirus HPV-DNA with high-grade cervical intraepithelial neoplasia (CIN 2 or 3): the role of cigarette smoking. Sex Transm Infect 1999; 75:406-8.

25. Monsonego J, Bosch FX, Coursaget P, Cox JT, Franco E, Frazer I, et al. Cervical cancer control, priorities and new directions. Int J Cancer 2004; 108:329-33.

26. Eluf-Neto J, Nascimento CMR. Cervical cancer in Latin América. Semin Oncol 2001; 28:188-97.

27. Moreno V, Bosch FX, Munõs N, Meijer CJ, Shah $\mathrm{KV}$, Walboomers JM, et al. Effect of oral contraceptives on risk of cervical cancer in women with human pappilomavirus infection: the IARC multicentric case-control study. Lancet 2002; 359. 1085-92.

Recebido em 03/Set/2003

Versão final reapresentada em 18/Ago/2004

Aprovado em 30/Ago/2004 